\title{
Captura y almacenamiento de carbono como servicio ambiental en el bosque de q'euña "La Paz" Parque Arqueológico de Sacsayhuamán, Cusco
}

\author{
Carbon capture and storage as an environmental service in the Polylepis forest "La Paz", \\ Archaeological Park of Sacsayhuamán, Cusco
}

\author{
Isabel Rodríguez-Sánchez ${ }^{1 *}$, Violeta E. Zamalloa-Acurio ${ }^{I}$, María A Aguilar-Lopez ${ }^{1}$, \\ Vilma Champi-Ayma ${ }^{2}$ María E. Ezquerra-Condori ${ }^{3}$, \& Jonyer H. Zapata-Callañaupa ${ }^{1}$ \\ ${ }^{l}$ Escuela Profesional de Biología; Facultad de Ciencias. Universidad Nacional San Antonio Abad del Cusco, \\ Av. de la Cultura 733, Cusco- Perú \\ ${ }^{2}$ Gerencia de Recursos Naturales y Medio Ambiente. Gobierno Regional Cusco \\ 3Asociación Civil sin Fines de Lucro para la Biodiversidad, Investigación y Desarrollo en Ecosistemas Tropicales \\ *Autor corresponsal: Isabel Rodríguez_Sánchez,rod_isabel@yahoo.es
}

\begin{abstract}
RESUMEN
El presente estudio se realizó en el bosque de q'euña La Paz, ubicado en el Parque Arqueológico de Sacsayhuamán, distrito, provincia y región Cusco, en el año 2017, con la finalidad de evaluar la captura y almacenamiento de carbono como servicio ambiental, se utilizó el método cartográfico mediante el S.I.G. utilizando el Software Arcgis 10.4 y como instrumento el GPS, para la captura de carbono se aplicó el método estandarizado RAINFOR de estimación cuantitativa mediante las ecuaciones alométricas de Chave et. al 2005 y Sarcca 2017, empleadas para el cálculo de contenido de carbono en biomasa aérea y el método cuantitativo para determinar la valoración económica de captura y almacenamiento de carbono como servicio ambiental, llegando a las siguientes conclusiones, el área de bosque de Q'euña "La Paz" es de $31838 \mathrm{~m}^{2}$ respecto a la captura y almacenamiento de carbono de la biomasa aérea de árboles de Q'euña, presentan un stock de carbono total de 14.16 tnC utilizando la ecuación alométrica de Chave 2005 y 20.61 tnCha, utilizando la ecuación alométrica de Sarcca 2017, dde acuerdo a la valoración económica de la captura y almacenamiento de carbono de la biomasa aérea como servicio ambiental para el bosque de Polylepis asciende a 537.64 y 782.31 euros utilizando las ecuaciones alométricas de Chave 2005 y Sarcca 2017 respectivamente.
\end{abstract}

Palabras clave: Captura de carbono, servicio ambiental, stock de carbono.

The present study was conducted in the Polylepis La Paz forest, located in the Archaeological Park of Sacsayhuamán, district, province and Cusco region, in the year 2017, with the purpose of evaluating the capture and storage of carbon as an environmental service, the cartographic method was used by the SIG using the Arcgis Software 10.4 and as an instrument the GPS, for the capture of carbon, the standardized RAINFOR method of quantitative estimation was applied by the allometric equation of Chave et. to 2005 and Sarcca 2017 used for the calculation of carbon content in aerial biomass and the quantitative method to determine the economic valuation of carbon capture and storage as an environmental service, reaching the following conclusions, the forest area of Polylepis "La Paz" it is $31838 \mathrm{~m} 2$ with respect to the carbon capture and storage of the aerial biomass of Polylepis trees, they present a total carbon stock of $14.16 \mathrm{tnC}$ using the Chaome 2005 allometric equation and $20.61 \mathrm{tnC}$ using the equation alometrica de Sarcca 2017, according to the economic valuation of carbon capture and storage of aerial biomass as an environmental service for the Polylepis forest, amounts to 537.64 euros and 782.31 euros using the alometric equations of Chave 2005 and Sarcca 2017 respectively.

Key words: Carbon capture, environmental service, carbon stock.

\section{INTRODUCCIÓN}

La investigación en captura de carbono por sistemas naturales se encuentra relacionada con el estudio del valor de las funciones ecológicas de los ecosistemas naturales. En 1976 sale la idea que los bosques son "almacenadores" de las emisiones de combustibles fósiles específicamente el $\mathrm{CO} 2$ (WRI 2001).

Los bosques nativos son de gran importancia en las zonas andinas, ya que cumplen funciones importantes como la conservación de suelos, mejorando las condiciones y reciclaje de nutrientes del suelo, albergan a diferentes especies de animales, aumenta la temperatura generando un microclima dentro del sistema, fija el carbono y protege las cabeceras de cuenca, además de ellas se obtienen importantes productos maderables. En general incrementan las ventajas ambientales, sociales y económicas en la sociedad (Arica, 2005).

La presente investigación tuvo como finalidad determinar el área de bosque de Q'euña (Polylepis) "La Paz" localizado en el Parque Arqueológico de Sacsayhuamán, distrito, provincia y región Cusco; también se estimó la captura y almacenamiento de carbono de la biomasa aérea de árboles de q`euña (Polylepis) mediante las ecuaciones alométicas de Chave 2005 y Sarcca 2017 y se realizó la valoración económica de la captura y almacenamiento de carbono de la biomasa aérea como servicio ambiental. Los datos obtenidos servirán como base para futuros trabajos de investigación respecto al conocimiento de cuanto carbono almacena dicho bosque y porque es tan importante su mantenimiento, conservación y los beneficios ecosistémicos.

\section{METODOLOGÍA}

Determinación del área del bosque de q'euña

Se aplicó el método cartográfico mediante el Sistema de información geográfica - S.I.G. utilizando el Software Arcgis 10.4 y como instrumento el GPS para determinar las coordenadas UTM para elaborar la delimitación del área ocupada por el bosque materia de estudio. (Fig. 1)

Estimación de la captura y almacenamiento de carbono de la biomasa aérea

La determinación de las unidades muestrales se realizó mediante el muestreo aleatorio (Mateucci \& Colma, 1982). En 


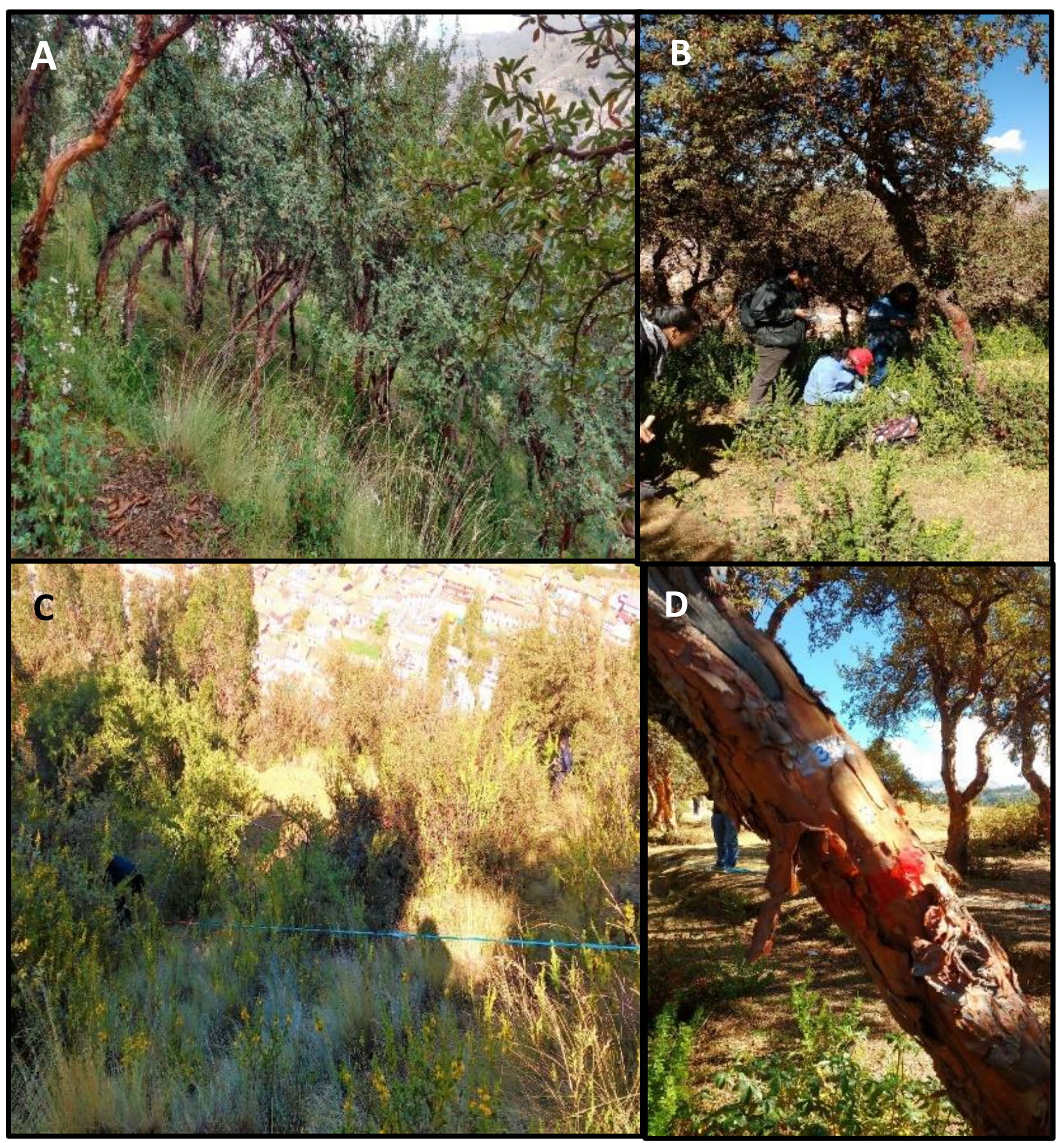

Figura 1: A. Bosque de q'euña La Paz - Sacsayhuamán, B. Diseño metodológico grupal, C. Delimitación del área de estudio, D. Determinación del DAP.

forma aleatoria se determinó 02 parcelas de 20 x 50m $\left(1000 \mathrm{~m}^{2}\right)$ considerando la accesibilidad y posibilidad de evaluar el área por completo. La estimación de la captura y almacenamiento de la biomasa aérea de carbono se realizó siguiendo lo establecido en el manual de RAINFOR.

\section{Ecuación alométrica de Chave (2005)}

Correspondiente a bosque secos de sierra para lo cual es necesario medir el depósito de vegetación arbórea mediante mediciones del DAP > $10 \mathrm{~cm}$ de diámetro y la altura del individuo.
Depósito en vegetación arbórea:

Ecuación / Factor Alcance Región Referencia (Mg de peso seco $\mathrm{ha}^{-1}$ )

$$
\text { BVA }=0,112 \times(\rho * D A P 2 * h t){ }^{0,916}
$$

0,64 densidad de madera por defecto (Chave et al. 2005; Zanne et al. 2009).

Dónde:

$\mathrm{BVA}=$ Biomasa de la vegetación arbórea.

$\rho \quad=$ Densidad de la madera.

DAP = Diámetro a la altura de pecho

ht $=$ altura del árbol 
Ecuación alométrica propuesta por Sarcca. 2017

Para el cálculo de la biomasa se aplicó la formula determinada por Sarcca 2017, para el género Polylepis:

$$
\mathrm{B}=0.16496[\mathrm{~A}+\mathrm{D}]^{2.667785}
$$

$\mathrm{B}=$ Biomasa de la vegetación arbórea.

$\mathrm{A}=$ altura del árbol

$\mathrm{D}=$ Diámetro de copa

\section{La densidad de carbono ( $\operatorname{tnC}$ ha -1$)$}

La densidad de carbono se calculó a partir de la siguiente relación (IPCC, 2006):

Densidad de Carbono $($ tnC ha -1$)=$ biomasa $/ 2$

\section{Stock de carbono}

El stock de carbono se calculó a partir de la siguiente relación:

Stock de carbono=Densidad de Carbono promedio (tnCha-1) $\mathrm{X}$ Extensión total

\section{Estimación del servicio ecosistémico}

Sobre la base de los pesos moleculares (44/12), una tonelada de carbono equivale a 3.67 toneladas de $\mathrm{CO} 2$-eq, (Fonseca, 2005). De esta forma, para saber la cantidad de CO2eq secuestrado y almacenado en la vegetación del bosque de Polylepis del bosque la Paz (servicio ecosistémico), se multiplicó la cantidad del stock de carbono por el valor numérico 3.67.

\section{CO2-eq $=$ Stock de carbono ecosistema X 3.67}

Dónde:

CO2-eq: Dióxido de carbono equivalente.

3.67: Factor de conversión

Valoración económica de la captura y almacenamiento de carbono de la biomasa aérea como servicio ambiental.

Se realizó con el método cuantitativo para determinar la valoración económica de captura y almacenamiento de carbono como servicio ambiental (Fonseca, 2005).

\section{Determinación de la valoración económica}

El método de valoración económica aplicado es el método de precios de mercado (MPM), donde la estimación económica del servicio ambiental de captura de carbono se realizó a través de la multiplicación del valor dado para la estimación ambiental y el valor monetario sugerido para la emisión de dióxido de carbono equivalente por cada tonelada.

Debido a que el $90 \%$ de las transacciones mundiales de derechos de emisión se realizan en Europa, se vio por conveniente utilizar los precios de emitir una tonelada de $\mathrm{CO} 2$ en Europa. El precio que se utilizó es el promedio de las medias anuales desde el año 2008 hasta el 2016 (Tabla 1).

La ecuación que se utilizó para calcular el valor económico del servicio ecosistémico es el siguiente:

$\mathrm{VE}=\mathrm{t} \mathrm{CO}_{2} \times \mathrm{P}$

Dónde:

t $\mathrm{CO}_{2}=$ Toneladas de dióxido de carbono equivalente.

$\mathrm{P}=$ Precio utilizado para cada tonelada de $\mathrm{CO} 2$.

\section{RESULTADOS Y DISCUSION}

Área del bosque de q'euña localizado en el Parque Arqueológico de Sacsayhuamán

El proyecto de investigación contempla como área de estudio 3.1838 ha correspondiente al bosque de Polylepis del bosque La Paz ubicado en el Parque Arqueológico de Sacsayhuamán (Fig. 2).

Captura y almacenamiento de carbono de la biomasa aérea

Se muestra los resultados de las mediciones dasométricas de los árboles de Q'euña pertenecientes a la parcela 1, así como la cantidad de almacenamiento de carbono de la biomasa aérea de cada árbol. El total de biomasa obtenida en la parcela 1 es de $9 \mathrm{tnC} / \mathrm{ha}$ y $12.24 \mathrm{tnC} / \mathrm{ha}$; utilizando las ecuaciones alométricas de Chave y Sarcca respectivamente, y la densidad de biomasa con la ecuación alométricas de Chave es de 4.50 tnC/ha y con la ecuación alométrica Sarcca es 6.12 tnC/ha (Anexo 1).

Se muestra los resultados de las mediciones dasométricas de los árboles de q’euña pertenecientes a la parcela 2, así como la cantidad de almacenamiento de carbono de la biomasa aérea de cada árbol. El total de biomasa obtenida en la parcela 2, es de $8.80 \mathrm{tnC} / \mathrm{ha}$ y $13.66 \mathrm{tnC} / \mathrm{ha}$; utilizando las ecuaciones alométricas de Chave y Sarcca respectivamente, y la densidad de biomasa con la ecuación alométrica de Chave es de 4.39 tnC/ha y con la ecuación alométrica Sarcca es $6.83 \mathrm{tnC} / \mathrm{ha}$ (Anexo 2).

La biomasa obtenida utilizando la ecuación alométrica de Chave es, en algunos casos, de menor valor comparándolo a lo obtenido por la ecuación alométrica de Sarcca, dicha variación podría estar relacionado al ámbito de estudio de Chave, quién determinó sus ecuaciones en base a investigaciones realizadas en bosques netamente tropicales donde la morfología y densidad de madera es muy diferente a la de Polylepis. Sin embargo, cabe resaltar que en el presente estudio se optó por el uso de estas ecuaciones alométricas con el fin de evitar el uso de métodos destructivos que dañen directamente el bosque de q'euña (Polylepis) que se encuentran comprendidos en una zona protegida como patrimonio cultural de la humanidad.

Los valores determinados en el presente estudio mediante la ecuación alométrica de Sarcca en las parcelas 1 y 2 (Anexos 1 y 2) concuerdan con los hallados por Mansilla, $\mathrm{H}$ en el 2012 y Sarcca, en el 2017, lo cual se podría explicar porque ambos autores estimaron la densidad de carbono para un bosque de Polylepis.

A partir del valor de la densidad de biomasa promedio de las parcelas 1 y 2 se tiene que el stock carbono obtenido para el bosque de Polylepis es de 14.167 tnC y de 20.61 tnC según las ecuaciones alométricas de Chave 2005 y Sarcca 2017 (Tabla 2).

Tabla 1: Precios de tonelada (tn) CO2 - equivalencia (EQ) de las medias anuales en euros ( $\epsilon$ )

\begin{tabular}{lccccccccc}
\hline Año & $\mathbf{2 0 1 6}$ & $\mathbf{2 0 1 5}$ & $\mathbf{2 0 1 4}$ & $\mathbf{2 0 1 3}$ & $\mathbf{2 0 1 2}$ & $\mathbf{2 0 1 1}$ & $\mathbf{2 0 1 0}$ & $\mathbf{2 0 0 9}$ & $\mathbf{2 0 0 8}$ \\
\hline Precios & $5.35 €$ & $7.68 €$ & $5.96 €$ & $4.45 €$ & $7.33 €$ & $12.89 €$ & $14.32 €$ & $13.06 €$ & $22.02 €$
\end{tabular}



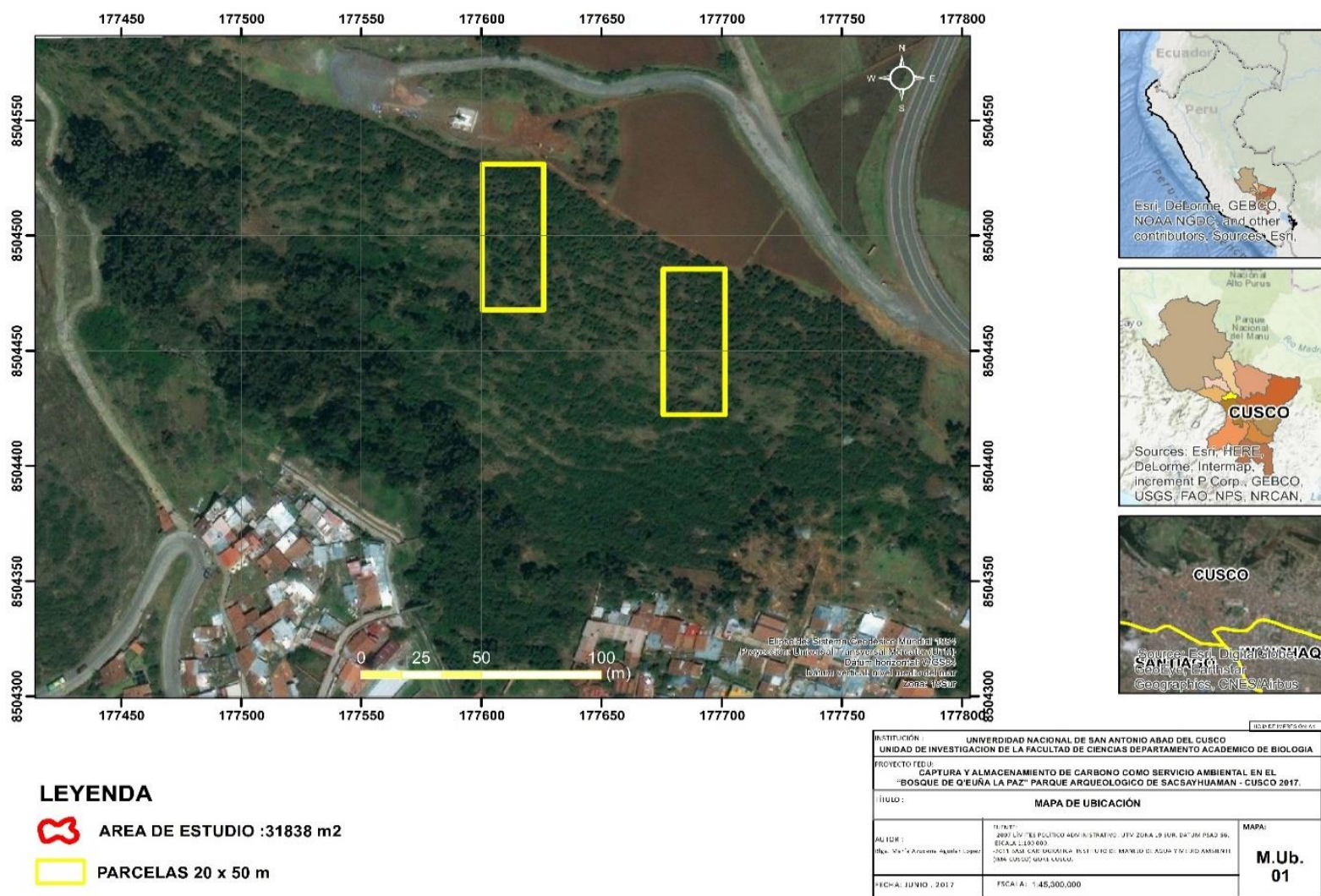

\section{LEYENDA}

3 AREA DE ESTUDIO : $31838 \mathrm{~m} 2$

PARCELAS $20 \times 50 \mathrm{~m}$
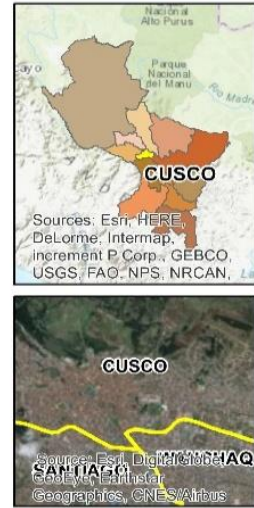

Figura 2. Mapa de la ubicación del bosque de q'euña (Polylepis) "La Paz” del Parque Arqueológico de Sacsayhuamán

Tabla 2: Estimación del stock de carbono

\begin{tabular}{lcc}
\hline \multicolumn{1}{c}{ Stock de Carbono } & $\begin{array}{c}\text { AGB(tnC) } \\
\text { Chave, 2005 }\end{array}$ & $\begin{array}{c}\text { AGB (tnC) } \\
\text { Sarcca, 2017 }\end{array}$ \\
\hline $\begin{array}{l}\text { Densidad de biomasa } \\
\text { promedio }\end{array}$ & 4.45002464 & 6.47510691 \\
Stock de carbono total & 14.1679884 & 20.6154454 \\
\hline
\end{tabular}

Tabla 3: Estimación del servicio ecosistémico

\begin{tabular}{ccc}
\hline $\begin{array}{c}\text { Servicio } \\
\text { ecosistémico }\end{array}$ & AGB(Kg) Chave 2005 & $\begin{array}{c}\text { AGB (kg) } \\
\text { Sarcca. 2017 }\end{array}$ \\
\hline CO2-eq & 51.99651758 & 75.6586846 \\
\hline
\end{tabular}

Tabla 4: Valor económico del servicio ecosistémico de secuestro y almacenamiento de carbono para el bosque de Polylepis.

\begin{tabular}{lcc}
\hline $\begin{array}{l}\text { Valor económico del } \\
\text { servicio ecosistémico }\end{array}$ & $\begin{array}{c}\text { AGB(Kg) } \\
\text { Chave 2005 }\end{array}$ & $\begin{array}{c}\text { AGB (kg) } \\
\text { Sarcca 2017 }\end{array}$ \\
\hline CO2-eq & 51.996518 & 75.6586685 \\
VE $(\boldsymbol{\epsilon})$ & 537.65399 & 728.3108 \\
\hline
\end{tabular}

Valoración económica de la captura y almacenamiento de carbono de la biomasa aérea como servicio ambiental

La cantidad de carbono equivalente es de 51.996 y 75.658 según las ecuaciones alométricas de Chave 2005 y Sarcca 2017 respectivamente (Tabla 3 ).
Valoración económica del servicio ecosistémico

A continuación, se muestra las fases de la valoración económica resueltas (Fonseca, 2005):

\section{Preparación de la Valoración eConómica}

\section{a. Identificación del objetivo de la valoración}

Actualmente el bosque de Polylepis sufre presión antrópica como contaminación por residuos sólidos; es por ello que es necesario implementar programas y/o proyectos que impulsen la reducción de emisiones por deforestación y degradación, la conservación y aumento de las reservas de carbono forestal con especies nativas y la gestión sostenible del bosque.

\section{b. Definición del ámbito}

Bosque de q'euña (Polylepis) La Paz, Parque Arqueológico de Sacsayhuamán.

\section{c. Identificación de los bienes y servicios ambientales}

Regulación hídrica, recreación, diversidad biológica, regulación de la erosión, siendo de importancia en la investigación el servicio ecosistémico, secuestro y almacenamiento de carbono.

\section{VALORACIÓN ECONÓMICA}

a. Identificación y caracterización de los actores involucrados

Los actores involucrados en el presente trabajo de investigación son el Ministerio de Cultura, debido a que el área de estudio pertenece al Parque Arqueológico de Sacsayhuamán y es jurisdicción de la Municipalidad Provincial del Cusco. 


\section{b. Priorización y caracterización de los bienes y servicios ecosistémicos}

El servicio ecosistémico priorizado del bosque de q'euña (Polylepis) La Paz, constituye el de secuestro y almacenamiento de carbono. El cual constituye un mecanismo de adaptación frente al cambio climático.

\section{c. Identificación de los tipos de valor económico}

El servicio ecosistémico de captura de carbono es un valor de uso indirecto; porque los beneficios del servicio no son exclusivos de un individuo en particular, sino que se extienden hacia la población adyacente al área de estudio; sin embargo, el servicio ecosistémico puede tener un valor de uso directo; porque los individuos y la sociedad en general obtienen beneficios por el uso de este servicio, en términos de mitigación al cambio climático.

\section{d. Elección y aplicación de los métodos de valoración económica}

La valoración económica, se determinó a partir del método de Precios de mercado (MPM); porque el servicio ecosistémico es transado en un mercado (mercado de los bonos de carbono) y porque las externalidades son mínimas. Además, se tiene información de precios, cantidades y costos.

El valor económico del servicio ecosistémico de secuestro y almacenamiento de carbono para el bosque de Polylepis asciende a 537.65 euros y 728.31 euros utilizando las ecuaciones alométricas de Chave y Sarcca respectivamente. Probablemente su valor ascienda por encontrarse dentro de un parque arqueológico (Tabla 6).

\section{Post VAloración ECONÓMICA}

\section{a. Aplicación del valor económico en la toma de decisiones}

La información generada en la valoración económica del servicio ecosistémico puede ser utilizada en la planificación de proyectos de conservación y gestión sostenible, en contabilidad regional, mecanismos de financiamiento (los mecanismos de retribución por servicios ecosistémicos y las transferencias directas condicionadas TDC), entre otros; a fin de frenar los procesos de deforestación y degradación en este bosque.

\section{b. Análisis del aporte a la solución del problema.}

Cuando se haya tomado las decisiones de acuerdo a la información generada aquí, se podrá evaluar y analizar el aporte a la solución del problema.

\section{CONCLUSIONES}

El área de bosque de q'euña (Polylepis) "La Paz" localizado en el Parque Arqueológico de Sacsayhuamán determinado en el presente estudio es de 3.1838 ha.

La captura y almacenamiento de carbono de la biomasa aérea de árboles de Q`euña, presentan un stock de carbono total de 14.17 tnC utilizando la ecuación alométrica de Chave 2005 y 20.61 tnC utilizando la ecuación alométrica de Sarcca 2017.

La valoración económica de la captura y almacenamiento de carbono de la biomasa aérea como servicio ambiental para el bosque de Q'euña (Polylepis) asciende a 537.64 euros y 782.31 euros utilizando las ecuaciones alométrica de Chave 2005 y Sarcca 2017 respectivamente. Probablemente su valor ascienda por encontrarse dentro del Parque Arqueológico de Sacsayhuamán.

\section{AGRADECIMIENTOS}

A los estudiantes del área de Ecología de la Escuela Profesional de Biológica - Facultad de Ciencias de la UNSAAC por su colaboración.

\section{REFERENCIAS BIBLIOGRÁFICAS}

Arica, D. (2005). Algunas especies forestales nativas para la zona alto andina. Perú. Consorcio para el desarrollo de la Ecorregión Andina, CONDESAN. 11 p.

Chave J., Andalo, C., Brown, S., et al. (2005) Tree Allometry and improved estimation of carbon stocks and balance in tropical forests. Oecología, 145, 87-99.

CIMA (2014). Monitoreo de Diversidad Vegetal y carbono en bosques andinos Consorcio para el Desarrollo Sostenible de la Ecorregión Andina (CONDESAN) Instituto de Ecología Regional de la Universidad de Tucumán. Agencia Suiza para el Desarrollo y la Cooperación (COSUDE) Programas Globales en los Andes Serie de protocolos de monitoreo. Protocolo 2.

Fonseca, W. (2005). Captura de carbono, diversidad y rentabilidad financiera en restauraciones activas y pasivas de bosque húmedo tropical en Costa Rica. Costa Rica.

González, M.J. (2011) Facultad de Ciencias Forestales y Conservación de la Naturaleza. Recuperado: http://www.forestal.uchile.cl/noticias/77673/la-importanciade-los-bosques-peri-urbanos.

Mansilla, H. (2001) Aspectos económicos de la captura de carbono en especies nativas: caso Bosque de Queuña Qocha. Ollantaytambo, Cuzco. Perú. En: Valoración Económica de la Diversidad Biológica y Servicios Ambientales del proyecto Conservación y Manejo de la Biodiversidad y Ecosistemas Frágiles- BIOFOR, pp. 407-438.

Sarcca, Y. (2017). Valoración económica del servicio ecosistémico de secuestro y almacenamiento de carbono en el bosque de Polylepis del Pichu Pichu, Arequipa. Tesis de Ingeniero Ambiental, Universidad Nacional de San Agustín de Arequipa.

Zanne A.E., López G.G., Comes D.A., Jansen S.J. \& Lewis, S.L. (2009). Base de datos de Densidad Global de Maderas. IIAP, Lima-Perú.

WRI (2001). Evaluating carbon sequestration projects: A first attempt. World Resources Institute, Washington DC. Disponible en: www.wri.org. 
Anexo 1: Mediciones dasométricas de árboles de q'euña (Polylepis), parcela $N^{\circ} 1$.

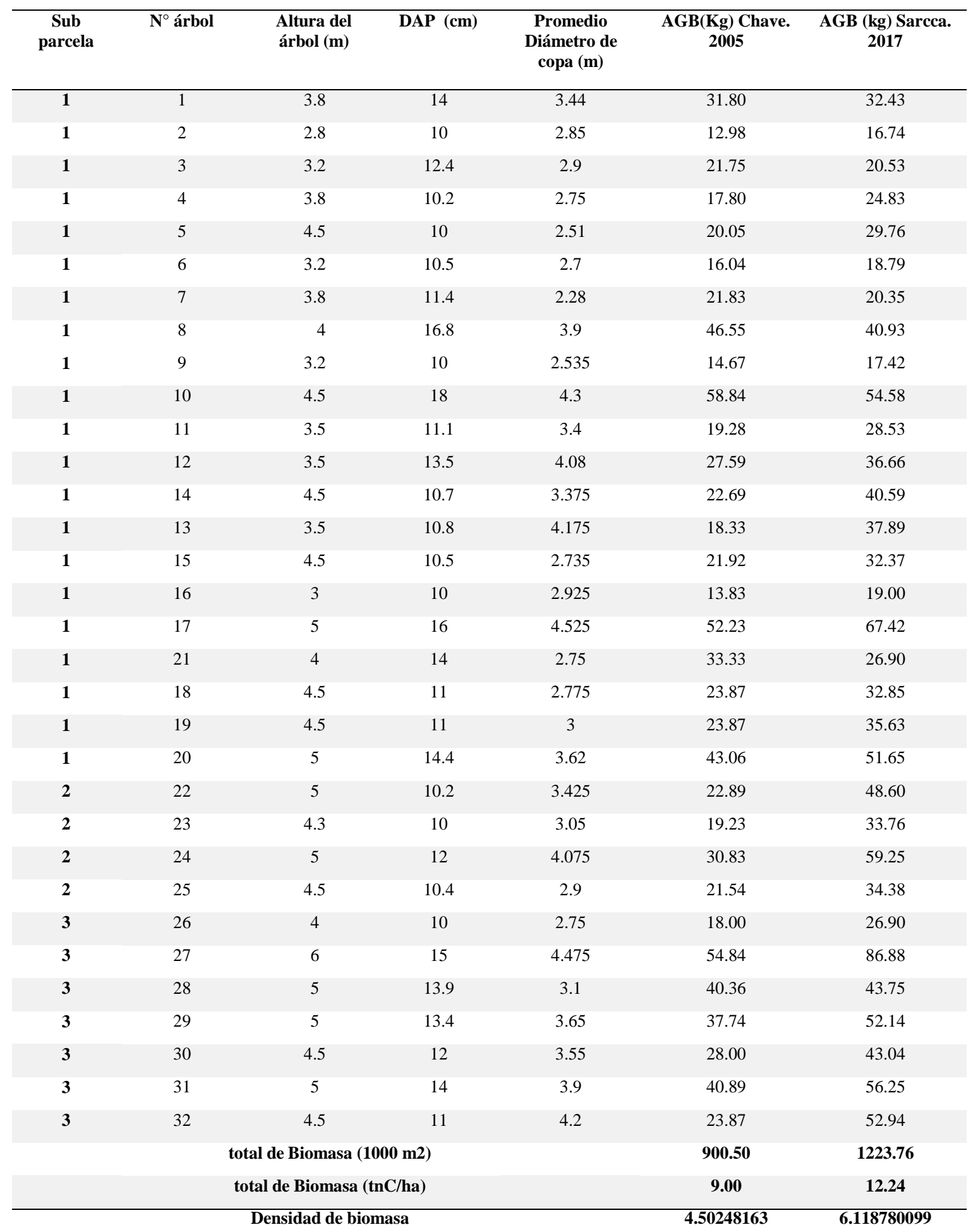


Anexo 2: Mediciones dasométricas de árboles de q'euña (Polylepis), parcela $N^{\circ} 2$

\begin{tabular}{|c|c|c|c|c|c|c|}
\hline $\begin{array}{c}\text { Sub } \\
\text { parcela }\end{array}$ & $\mathbf{N}^{\circ}$ árbol & $\begin{array}{l}\text { Altura del } \\
\text { árbol (m) }\end{array}$ & DAP (cm) & $\begin{array}{c}\text { Promedio Diámetro de } \\
\text { copa (m) }\end{array}$ & $\begin{array}{c}\text { AGB(Kg) } \\
\text { Chave,2005 }\end{array}$ & $\begin{array}{c}\text { AGB (kg) Sarcca, } \\
2016\end{array}$ \\
\hline 1 & 1 & 4 & 10.8 & 2.2 & 20.72 & 21.44 \\
\hline 1 & 2 & 4.5 & 12.2 & 2.85 & 28.86 & 33.76 \\
\hline 1 & 3 & 4.5 & 10.5 & 3.1 & 21.92 & 36.91 \\
\hline 1 & 4 & 3.7 & 12.6 & 3.2 & 25.59 & 28.53 \\
\hline 1 & 5 & 3.5 & 10 & 2.375 & 15.92 & 18.57 \\
\hline 1 & 6 & 4.5 & 10.7 & 3.4 & 22.69 & 40.93 \\
\hline 1 & 7 & 3.7 & 11.2 & 2.9 & 20.62 & 25.34 \\
\hline 1 & 8 & 5 & 11.7 & 2.55 & 29.43 & 36.27 \\
\hline 1 & 9 & 3.7 & 15.6 & 3.85 & 37.84 & 36.27 \\
\hline 1 & 10 & 3.7 & 13 & 2.575 & 27.10 & 22.14 \\
\hline 1 & 11 & 4.5 & 10.3 & 3 & 21.16 & 35.63 \\
\hline 1 & 12 & 4.3 & 10.2 & 3.05 & 19.94 & 33.76 \\
\hline 1 & 13 & 3.8 & 10.3 & 2.2 & 18.13 & 19.65 \\
\hline 1 & 14 & 4 & 11.9 & 3.1 & 24.75 & 30.79 \\
\hline 1 & 15 & 4.8 & 10.7 & 2.95 & 24.07 & 38.89 \\
\hline 1 & 16 & 4 & 12.2 & 3.55 & 25.90 & 36.27 \\
\hline 1 & 17 & 3.5 & 12.1 & 2.5 & 22.58 & 19.65 \\
\hline 1 & 18 & 4.5 & 10.1 & 2.95 & 20.41 & 35.00 \\
\hline 1 & 19 & 5 & 12.3 & 2.9 & 32.26 & 40.93 \\
\hline 2 & 20 & 4.2 & 12 & 13.35 & 26.28 & 344.23 \\
\hline 2 & 21 & 4 & 19 & 4.25 & 58.32 & 45.95 \\
\hline 2 & 22 & 3.5 & 11.8 & 2.35 & 21.56 & 18.36 \\
\hline 2 & 23 & 4.5 & 12.8 & 2.6 & 31.51 & 30.79 \\
\hline 2 & 24 & 3 & 14.3 & 3.65 & 26.63 & 25.85 \\
\hline 2 & 25 & 3.8 & 12.7 & 3.025 & 26.60 & 27.71 \\
\hline 2 & 26 & 3.2 & 11.3 & 3.7 & 18.35 & 28.53 \\
\hline 2 & 27 & 5 & 11 & 3.25 & 26.29 & 45.95 \\
\hline 2 & 28 & 3.2 & 10.1 & 3.05 & 14.94 & 21.91 \\
\hline 2 & 29 & 4 & 13 & 3.7 & 29.10 & 38.22 \\
\hline 2 & 30 & 3 & 10.5 & 2.45 & 15.12 & 15.20 \\
\hline 3 & 31 & 3.5 & 11.9 & 3.45 & 21.90 & 29.08 \\
\hline 3 & 32 & 2.8 & 10.1 & 3.05 & 13.22 & 18.36 \\
\hline 3 & 33 & 4 & 11.6 & 2.1 & 23.62 & 20.53 \\
\hline 3 & 34 & 3.8 & 10.1 & 2.925 & 17.49 & 26.64 \\
\hline 3 & 35 & 5 & 15.4 & 2.7 & 48.69 & 38.22 \\
\hline \multicolumn{5}{|c|}{ Total de Biomasa (1000 m2) } & 879.51 & 1366.29 \\
\hline \multicolumn{5}{|c|}{ Total de Biomasa (tnC/ha) } & 8.79513527 & 13.6628675 \\
\hline \multicolumn{5}{|c|}{ Densidad de biomasa } & 4.39756764 & 6.83143373 \\
\hline
\end{tabular}

